\title{
Broad Histogram Method for Multiparametric Hamiltonians
}

\author{
A. R. Lima[њ], P. M. C. de Oliveira and T. J. P. Penna \\ Instituto de Física, Universidade Federal Fluminense \\ Av. Litorânea, s/n - 24210-340 Niterói, RJ, Brazil \\ Last correction: December 7, 1999 - Printed: August 7, 2013
}

\begin{abstract}
We extended the Broad Histogram Method in order to obtain spectral degeneracies for systems with multiparametric Hamiltonians. As examples we obtained the critical lines for the square lattice Ising model with nearest and next-nearest neighbor interactions and the antiferromagnetic Ising model in an external field. For each system, the entire critical line is obtained using data from a single computer run. We also discuss the accuracy and efficiency of our method.
\end{abstract}

PACS numbers: 02.70.Lq, 05.50.+q, 75.10.Hk

The search for new and more efficient methods for computer simulations is always an intensive task in science. Multispin coding techniques (see [1] and references therein), cluster algorithms [2, 3, 团, reweighting procedures [5, 6, 7] and methods which calculate directly the spectral degeneracy [8, 9, 10, 11] are a few remarkable examples (see [12, 13, 14] for reviews). An efficient implementation of these methods can lead to an enormous increase in speed and accuracy of computer simulations. Multiparametric simulation is another way to further improve the efficiency. Multiparametric simulations allow, from a single computer run, to explore the whole space of parameters defining a given system, instead of repeating the whole process every time some parameter is changed.

For single-parameter simulations, several methods such as the histogram method [5], simulated tempering [7], multicanonical ensemble [8, 9], 1/k-sampling [10], broad histogram [11] among others have been shown to be successful. On the other hand, only a few works in the literature treat multiparametric simulations using such methods. The histogram method [6] is easily generalized to any number of parameters of the Hamiltonian. However the main problem in this approach is that the number of simulations needed to cover a given region in the space of parameters increases 
with the system size, in a $d$-dimensional space, as $L^{d / 2}$ for each parameter 6 . Shteto et al. used the multicanonical method (Entropic Sampling formulation) in order to study relaxation paths in the simulation of an Ising model in an external field [15]. In that work they pointed out that the entropic sampling can be generalized to any number of parameters. Although easily generalizable, a simple implementation of this method has been shown to display a very slow convergence [14, 16]. On the other hand, methods such as the traditional multicanonical formulation [8] seem to be not easily extendable to perform simulations with more than one parameter. Recently a re-formulation of the broad histogram method was introduced including an external field as a second parameter [17.

In this paper we perform a spectral degeneracy calculation by applying the Broad Histogram method (BHM) to multiparametric Hamiltonians. We show that the method can be generalized to any number of parameters and the full range of them can be exploited. BHM was introduced three years ago [11] and it is based on an exact relation between the spectral degeneracy and some special macroscopic quantities defined within the method itself. A remarkable feature of BHM is its generality, since it is not restricted to any specific dynamical rule: the macroscopic quantities needed can be obtained by different procedures. BHM has been applied to a variety of magnetic systems with accurate results in a very efficient way [11, 16, 17, 18, 19, 20, 21, 22, 23.

Any statistical system can be described by two different classes of parameters: the ones which characterize it and are defined in the Hamiltonian, and the others which control the interaction with the environment. Let us consider the Hamiltonian of a given system as composed by $P$ independent interaction terms

$$
\mathcal{H}(\mathbf{X})=\gamma_{1} \mathcal{S}_{1}(\mathbf{X})+\gamma_{2} \mathcal{S}_{2}(\mathbf{X})+\ldots+\gamma_{P} \mathcal{S}_{P}(\mathbf{X})
$$

where the terms $\mathcal{S}_{1}, \ldots, \mathcal{S}_{P}$ depends only on the microstate $\mathbf{X}$ of the system. $\gamma_{1}, \ldots, \gamma_{P}$ are the parameters associated to these terms and all together define the complete Hamiltonian. For instance, $\mathcal{S}_{1}$ could represent the coupling with an external field, $\mathcal{S}_{2}$ a first-neighbor interaction, and so on. The temperature, on the other hand, belongs to the class of parameters which determines the interaction with the environment.

Before presenting the method, it is fundamental to introduce the concept of macrostate. To a given state $\mathbf{X}$ of the system, each $\mathcal{S}_{i}(\mathbf{X})$ gives a value $s_{i}$. These values define a vector $\mathbf{s}=$ 
$\left(s_{1}, s_{2}, \ldots, s_{P}\right)$. Hence, the set of all states $\mathbf{X}$ corresponding to the same $\mathbf{s}$ is called a macrostate. The space defined by all macrostates is called space of macrostates. The interaction with the environment corresponds to the choice of the statistical ensemble, described by a weight function $f\left(\mathcal{H}, \alpha_{1}, \ldots, \alpha_{P_{f}}\right) \equiv f\left(\gamma_{1}, \ldots, \gamma_{P}, \alpha_{1}, \ldots, \alpha_{P_{f}}, \mathbf{s}\right)$ whose functional form is known a priori. The control parameters for such interaction are $\alpha_{1}, \ldots, \alpha_{P_{f}}$. Once the system (characterized by $\mathbf{s}, \gamma_{1}, \ldots, \gamma_{P}$ ) and its interaction with the environment (characterized by $\alpha_{1}, \ldots, \alpha_{P_{f}}$ ) are defined, the thermodynamic average of any quantity $Q$, can be written as

$$
\langle Q\rangle_{\alpha_{1}, \ldots, \alpha_{P_{f}}}=\frac{\sum_{\mathbf{s}} g(\mathbf{s})\langle Q(\mathbf{s})\rangle f\left(\gamma_{1}, \ldots, \gamma_{P}, \alpha_{1}, \ldots, \alpha_{P_{f}}, \mathbf{s}\right)}{\sum_{\mathbf{s}} g(\mathbf{s}) f\left(\gamma_{1}, \ldots, \gamma_{P}, \alpha_{1}, \ldots, \alpha_{P_{f}}, \mathbf{s}\right)}
$$

where $g(\mathbf{s})$ is the spectral degeneracy, i.e. the number of possible microstates of the system corresponding to the same macrostate $\mathbf{s} .\langle Q(\mathbf{s})\rangle$ is the uniform average of $Q$ in the macrostate s. The parameters introduced by $f$ are irrelevant for the definition of $\mathbf{s}$ and consequently to the calculation of $g(\mathbf{s})$ (this idea has already been used in another context [18, 24]). Also, $g(\mathbf{s})$ is independent of the particular values $\left(\gamma_{1}, \ldots, \gamma_{P}\right)$ of the Hamiltonian parameters. Therefore, if one is able to provide the spectral degeneracy $g(\mathbf{s})$ and $\langle Q(\mathbf{s})\rangle$, all information about the observable $Q$ can be obtained for the full range of the parameters $\left(\gamma_{1}, \ldots, \gamma_{P}, \alpha_{1}, \ldots, \alpha_{P_{f}}\right)$.

In our generalization of BHM the spectral degeneracy is calculated through the steps:

Step 1: Choice of a reversible protocol of allowed movements in the space of microstates. Reversible protocol means that each allowed movement $\mathbf{X}_{\text {old }} \rightarrow \mathbf{X}_{\text {new }}$ corresponds to another also allowed movement $\mathbf{X}_{\text {new }} \rightarrow \mathbf{X}_{\text {old }}$. This definition is independent of the probabilities prescribed to these movements by the particular dynamics to be used in a practical computer implementation. These movements are virtual ones, since they are not actually performed.

Step 2: To compute the number $N(\mathbf{X}, \boldsymbol{\Delta} \mathbf{s})$ of allowed movements, for a configuration $\mathbf{X}$, that changes the current macrostate $\mathbf{s}$ to another $\mathbf{s}+\boldsymbol{\Delta} \mathbf{s}$. Then $\langle N(\mathbf{s}, \boldsymbol{\Delta} \mathbf{s})\rangle$ is the average of $N(\mathbf{X}, \boldsymbol{\Delta} \mathbf{s})$, i.e., the mean number of possible movements from macrostate $\mathbf{s}$ to macrostate $\mathbf{s}+\boldsymbol{\Delta} \mathbf{s}$;

Step 3: Due to the reversibility condition (step 1), the total number of possible movements from macrostate $\mathbf{s}+\boldsymbol{\Delta} \mathbf{s}$ to macrostate $\mathbf{s}$ is equal to the total number of possible movements from macrostate $\mathbf{s}$ to macrostate $\mathbf{s}+\Delta \mathbf{s}$. Thus, we can write down the equation

$$
g(\mathbf{s})\langle N(\mathbf{s}, \boldsymbol{\Delta} \mathbf{s})\rangle=g(\mathbf{s}+\mathbf{\Delta} \mathbf{s})\langle N(\mathbf{s}+\mathbf{\Delta} \mathbf{s},-\mathbf{\Delta} \mathbf{s})\rangle .
$$


This relation has been shown to be exact for any statistical model and spectrum degeneracy [19]. Our multiparametric formulation remain valid based on the same arguments. Therefore, equation (3) can be rewritten as

$$
\ln g(\mathbf{s}+\boldsymbol{\Delta} \mathbf{s})-\ln g(\mathbf{s})=\ln \frac{\langle N(\mathbf{s}, \boldsymbol{\Delta} \mathbf{s})\rangle}{\langle N(\mathbf{s}+\boldsymbol{\Delta} \mathbf{s},-\boldsymbol{\Delta} \mathbf{s})\rangle} .
$$

This equation can be iteratively solved for all macrostates $\mathbf{s}$, provided $\langle N(\mathbf{s}, \boldsymbol{\Delta} \mathbf{s})\rangle$ is known (again, this relation is independent of the way $\langle N(\mathbf{s}, \Delta \mathbf{s})\rangle$ is obtained). This set of equations is overdetermined. However, the spectral degeneracy can be obtained without solving all equations simultaneously, since all values of the projections of $\Delta \mathbf{s}$ along a given direction of the space of macrostates are equivalent.

The Broad Histogram relation (3) is independent of the procedure by which $\langle N(\mathbf{s}, \Delta \mathbf{s})\rangle$ is obtained. For the single parameter case, virtually any procedure can be adopted in this task as, for instance, a non-biased random walk [11], a microcanonical simulation [20] or the random walk performed by the Entropic Sampling in the space of microstates [16]. For the multiparametric case, the choice of the dynamics is crucial to an efficient implementation, because the larger the number of parameters, the larger and sparser is the space of macrostates. In this work we choose a dynamics based on the Entropic Sampling method (ESM). Lima et al. [16] have shown that this dynamics associated to BHM gives better results than the traditional Multicanonical calculation. The dynamics is implemented as follows: first, we implement the classical algorithm of ESM, performing the visitation in the space of macrostates. Additionally, after a given number of random walk steps (in our case one Monte Carlo Step (MCS)), for each visited state $\mathbf{X}$, we store the values of $N(\mathbf{X}, \boldsymbol{\Delta} \mathbf{s})$ cumulatively into s-histograms. This dynamics is particularly interesting due to its simplicity. The spectral degeneracy is calculated by the BHM relation (3), instead, avoiding the shortcomings of the traditional determination of the spectral degeneracy by ESM.

The procedure described above is quite general and can be applied to any statistical model. As examples of applications of the method we choose two traditional multiparametric problems: i) The Ising model with nearest and next-nearest neighbor interactions and ii) the antiferromagnetic Ising model in an external field. Within the canonical ensemble, two parameters come from the Hamiltonian and one (the temperature $T$ ) from the ensemble definition.

We start describing our results for the Ising model with nearest and next-nearest neighbor 
interactions in a $L \times L$ square lattice. The Hamiltonian of this system is given by

$$
\begin{aligned}
\mathcal{H} & =-J_{1} \sum_{\mathrm{nn}} \sigma_{i} \sigma_{j}-J_{2} \sum_{\mathrm{nnn}} \sigma_{i} \sigma_{j} \\
& =-J_{1} E_{1}-J_{2} E_{2}
\end{aligned}
$$

where $\sigma_{i}= \pm 1$. The summations are carried over the nearest and next-nearest neighbors, respectively. Each macrostate is defined by the vector $\mathbf{s}=\left(E_{1}, E_{2}\right)$, which is independent of the set of parameters $J_{1}, J_{2}$ and $T$. The adopted protocol of movements is the single spin-flip. In this case, the allowed $\boldsymbol{\Delta}$ s are: $(-8,-8),(-8,-4),(-8,0),(-8,4),(-8,8), \ldots,(8,-8),(8,-4)$, $(8,0),(8,4),(8,8)$. For the implementation of the random walk in the space of macrostates within ESM, we need to define the number of entropy updates and the number of Monte Carlo steps (MCS) between each update. We choose the number of MCS between successive updates as increasing linearly with the number of updates already performed, such that when the process is finished, each macrostate is visited on average $\bar{v}$ times. The number of updates was chosen as 100 and $\bar{v}$ as 4000 . Fig. (1) presents the entropy $S\left(E_{1}, E_{2}\right)=\ln g\left(E_{1}, E_{2}\right)$ for a $10 \times 10$ system, obtained by solving (4) from the measured averages $\langle N(\mathbf{s}, \boldsymbol{\Delta} \mathbf{s})\rangle$. We have not used any additional procedure to optimize our simulations, as for example, to use the entropy obtained from another simulation as initial input for the Entropic Sampling-based random walk. We also choose to store informations for all possible values of $E_{1}$ and $E_{2}$. Once $g\left(E_{1}, E_{2}\right)$ is obtained, we can calculate any thermodynamical quantity, without resorting again to computer simulations. Of particular interest is the line defined by the critical temperature $T_{c}$ as a function of the ratio $K=J_{2} / J_{1}$. We have calculated the internal energy, magnetization, specific heat and magnetic susceptibility as continuous functions of both $K$ and $T$. We have used the temperature where the specific heat presents a maximum as an estimative to the critical point for each $K$. In figure (2), we present our results for different lattice sizes. The lines are normalized such that at $K=0, T_{c}=1$. In the same figure, we present the infinite lattice results obtained by Fan \& Wu using low-temperature series expansions 25]. Finite size effects are noticed only near to $K=-0.5$ (insets). An important advantage of our method is that it is possible to blow-up a given region in the space of parameters without need of additional runs. All points in the critical lines were calculated from the same $g\left(E_{1}, E_{2}\right)$ obtained from only one simulation for each size. Traditional approaches [26] need a new simulational run for each pair $(T, K)$. To our knowledge, the results presented here are the 
most detailed available. Shteto et al. [15 suggest $32 \times 32$ as the limit for their multiparametric simulations of the Ising model in an external magnetic field with Entropic Sampling. We have reached this limit with a much more elaborated problem.

As a second example, we choose to study the Ising model in an external magnetic field. The Hamiltonian of this system is given by:

$$
\begin{aligned}
\mathcal{H} & =-J \sum_{\mathrm{nn}} \sigma_{i} \sigma_{j}+h \sum_{\mathrm{i}} \sigma_{i} \\
& =-J E+h m
\end{aligned}
$$

where $\sigma_{i}= \pm 1$. The macrostates are defined by $\mathbf{s}=(E, m)$. Fig. (3) presents the entropy $S(E, m)$ for a $10 \times 10$ system. For this model, it is interesting to obtain the critical temperature as a function of the applied field, for the antiferromagnetic case $(J<0)$. In fig. (4) we present our results. The theoretical line obtained by Wang and Kim is also shown [27]. The agreement is remarkable for all values of the field. Again the pseudo-critical temperature was determined by the peak position in the specific heat. Due to the excellent agreement reached already with the sizes we have studied, we did not simulate larger lattices.

Concerning the CPU time necessary to perform such simulations, we present in table I the mean visitation on each macrostate, and the total CPU time. From these results the CPU time $\tau$ to obtain, in average, $\bar{v}$ visitations on each macrostate scales like $L^{a}$ where $a \approx 6$ as expected, since a factor $L^{d \times P}$ comes from the volume dependence of the space of macrostates and $L^{d}$ from the increase in the time to perform one Monte Carlo step. Spectral degeneracy calculation methods (as the BHM and Multicanonical) are not based on thermodynamical concepts and hence not subjected to critical slowing down, large energy barriers and other problems. Therefore, it is misleading to compare the CPU time of our method with other Monte Carlo canonical methods. Additionally, for traditional methods the larger the system the larger is the number of simulations at different temperatures one needs in order to determine the peak position for the specific heat. On the other hand, within the BHM once one already knows $g\left(E_{1}, E_{2}\right)(g(E, m))$, it is possible to obtain results for any values of $J_{1}, J_{2}, T(J, m, T)$ continuously. Other quantities, as the free energy, which are hard to obtain by traditional methods, can also be obtained from only one simulation. In this sense, our method introduces a huge computational speedup.

In conclusion, we have shown that the Broad Histogram Method can be extended to obtain 
spectral degeneracies for multiparametric Hamiltonians. This generalization allows us to calculate the thermodynamical averages for any set of the parameters which define the model continuously, from only one computer run. Conversely, traditional Monte Carlo simulations need to perform a new computer run each time any parameter of the system is changed. Compared with traditional Monte Carlo simulations, this feature of BHM represents an enormous computational gain. Moreover, the generalization is such that the method could be applied to any statistical model.

This work was partially supported by CNPq, CAPES and FAPERJ (Brazilian Agencies). ARL acknowledge S. L. A. de Queiroz for very useful discussions. 


\section{References}

[*] E-mail: arlima@if.uff.br

[1] de Oliveira P. M. C., Computing Boolean Statistical Models (World Scientific, 1991).

[2] Coniglio A. and Klein W., J. Phys. A13, 2775 (1980).

[3] Swendsen R. H., J. -S. Wang and Ferrenberg A. M., in The Monte Carlo Method in Condensed Matter Physics, ed. K. Binder, (Springer, Berlin) (1995).

Topics in Applied Physics 71, 75 (1992).

[4] Wolff U., Phys. Rev. Lett. 62, 361 (1989).

[5] Salzburg Z.W., Jacobson J.D. Fickett W. and Wood W.W., J. Chem. Phys., 30, 65 (1959).

[6] Ferrenberg A. M. and Swendsen R. H., Phys. Rev. Lett. 61, 2635 (1988).

Ferrenberg A. M. and Swendsen R. H., Phys. Rev. Lett 63, 1195 (1989).

Ferrenberg A. M. and Landau D. P., Phys. Rev. B44, 5081 (1991).

Ferrenberg A. M., Landau D. P. and Swendsen R. H., Phys. Rev. E51, 5092 (1995).

[7] Marinari E. and Parisi G., Europhys. Lett. 19, 451 (1992).

[8] Berg B. A. and T. Neuhaus, Phys. Lett. B267, 149 (1991). For a review see Int. J. Mod. Phys. C3, 1083 (1992).

[9] Lee J., Phys. Rev. Lett. 71, 211 (1993).

[10] Hesselbo B. and Stinchcombe R. B., Phys. Rev. Lett. 74, 2151 (1995).

[11] de Oliveira P. M. C., Penna T. J. P. and Herrmann H.J., Braz. J. Phys. 26, 677 (1996). Also in cond-mat/9610041.

de Oliveira P. M. C., Penna T. J. P. and Herrmann H.J., Eur. Phys. J. B1, 205 (1998).

de Oliveira P. M. C., in Computer Simulation Studies in Condensed Matter Physics XI, ed. D. P. Landau (Springer), 169, (1998).

[12] Marinari E. Optimized Monte Carlo Methods. Lectures given at the 1996 Budapest Summer School on Monte Carlo Methods, pag. 50, edited by J. Kertész and I. Kondor, Springer, Berlin-Heidelberg (1998). Available at cond-mat/9612010. 
[13] de Felício J. R. D. and Líbero V. L., Am. J. Phys. 64, 1281 (1996).

[14] Newman M. E. J. and Barkema G. T.. Monte Carlo Methods in Statistical Physics (Oxford University Press), (1999).

[15] Shteto I., Linares J. and Varret F., Phys. Rev. E56, 5128 (1997).

[16] Lima A. R., de Oliveira P. M. C. and Penna T. J. P., "Comparison between Broad Histogram and Multicanonical Methods", submitted to publication (1999).

[17] Kastner M., Muñoz J. D. and Promberger M., "Speeding Up Computer Simulations: The Transition Observable Method" in cond-mat/9906097.

[18] Lima A. R., Sá Martins J. S. and Penna T. J. P., Physica A268, 553 (1999).

[19] de Oliveira P. M. C., Eur. Phys. J. B6, 111 (1998).

[20] de Oliveira P. M. C., Int. J. Mod. Phys. C9, 497 (1998).

[21] de Oliveira P. M. C., Comp. Phys. Comm. 121-122, 16 (1999).

[22] Wang J. -S., Tay T. K. and Swendsen R. H., Phys. Rev. Lett 82, 476 (1999);

Wang J. -S., Eur. Phys. J. B8, 287-291 (1999);

"Monte Carlo algorithms based on the number of potential moves" in cond-mat/9903224.

[23] Muñoz J. D. and Herrmann H. J., Int. J. Mod. Phys. C10, 95 (1999);

Comp. Phys. Commun. 121-122, 13 (1999);

also in Computer Simulation Studies in Condensed-Matter Physics XII, edited by D.P. Landau (Springer,Berlin, in print) and cond-mat/9810292.

[24] Salazar R. and Toral R., "Scaling functions for Tsallis non-extensive statistics" in condmat/9906350. To appear in Phys. Rev. Lett.

[25] Fan C. and Wu F. Y., Phys. Rev 179, 560 (1969).

[26] Landau D. P., Phys. Rev. B21, 1285 (1980).

[27] Wang X.-Z. and Kim J. S., Phys. Rev. Lett. 78, 413 (1997). 


\section{FIGURE CAPTION}

Fig. 1 - Entropy for a $10 \times 10$ Ising model with nearest and next-nearest neighbor interactions. The axes represent the nearest $\left(E_{1}\right)$ and next-nearest neighbor $\left(E_{2}\right)$ terms of the corresponding Hamiltonian. The entropy does not cover the whole space because some pairs $\left(E_{1}, E_{2}\right)$ do not correspond to accessible states.

Fig. 2 - Comparison between simulations of the Ising model with nearest and next-nearest neighbor interactions (for $L \times L$ lattices) and low-temperature expansion [25] (solid line). The symbols correspond to $L$ equal to: 10 (empty circles), 16 (pluses), 20 (crosses), 32 (diamonds). We

also show some Metropolis results for $\mathrm{L}=32$ (filled circles). Finite size effects are more remarkable near to $K=-0.5$ (upper-left inset). In the other inset (down-right) we show the results obtained using the ferromagnetic susceptibility to determine the critical point, instead of the specific heat.

Fig. 3 - Entropy for a $10 \times 10$ Ising Model in a external magnetic field. The axes represent the spin coupling term $(E)$ and the external field term $(m)$.

Fig. 4 - Comparison between simulation and theoretical results for the antiferromagnetic Ising Model in a external magnetic field. The agreement between simulation and theoretical results is excellent. 


\section{TABLE}

\begin{tabular}{|c|c|c|c|}
\hline Model & L & Mean Visitation & CPU Time (s) \\
\hline$J_{1}, J_{2}$ & 10 & 4945.2 & 1191.6 \\
$J_{1}, J_{2}$ & 16 & 4537.2 & 22118.2 \\
$J_{1}, J_{2}$ & 20 & 4425.0 & 86137.8 \\
$J_{1}, J_{2}$ & 32 & 4277.4 & 1848197.1 \\
$J, h$ & 10 & 4761.4 & 1082.4 \\
$J, h$ & 20 & 4356.8 & 71002.0 \\
\hline
\end{tabular}

Table 1: CPU time and mean visitation for each lattice size. The simulations were carried out on a Dec Alpha 500. 
ن'

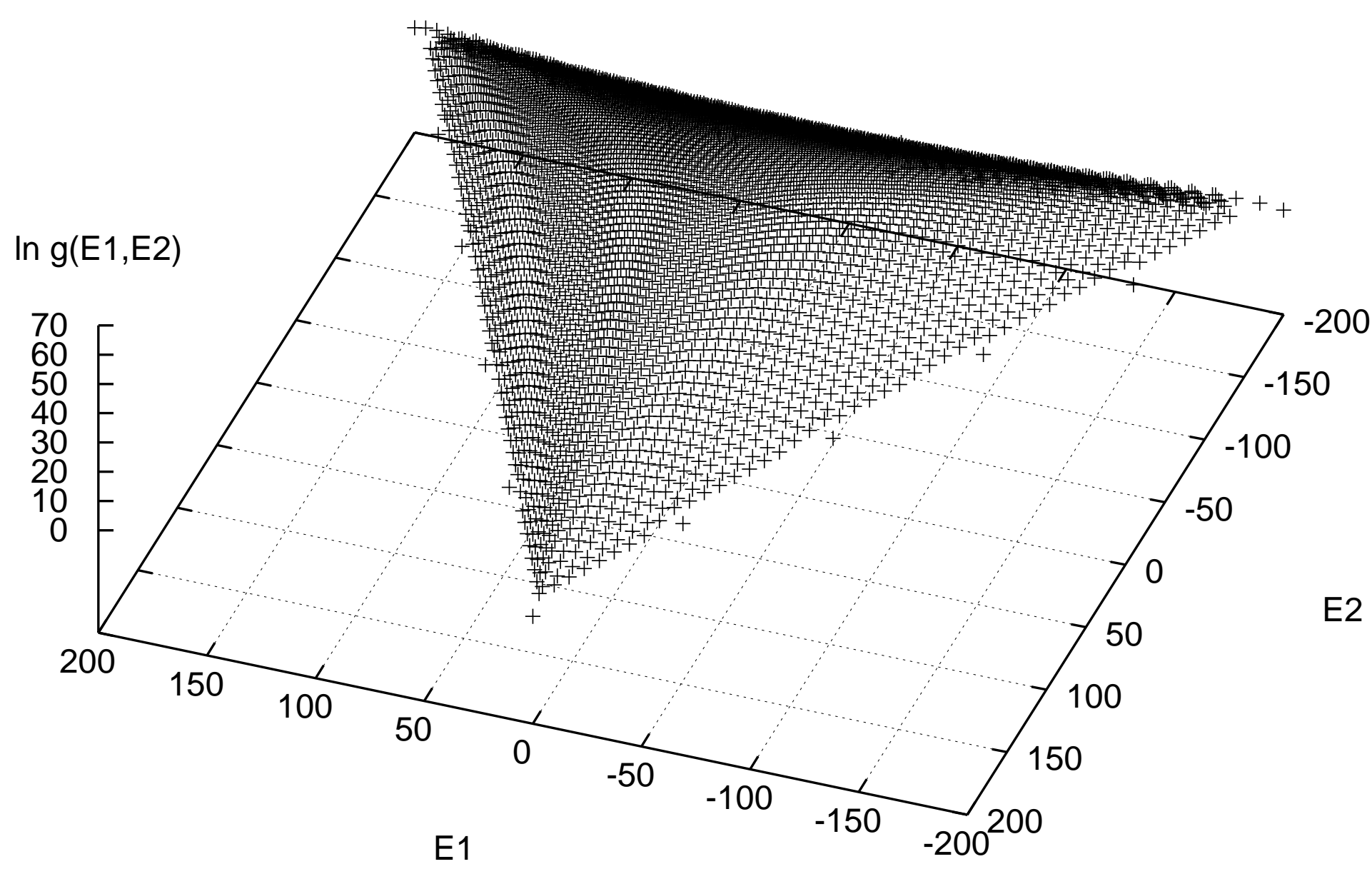


Fig. 2 - "Broad Histogram...", A.R. Lima, P.M.C. de Oliveira and T.J.P. Penna

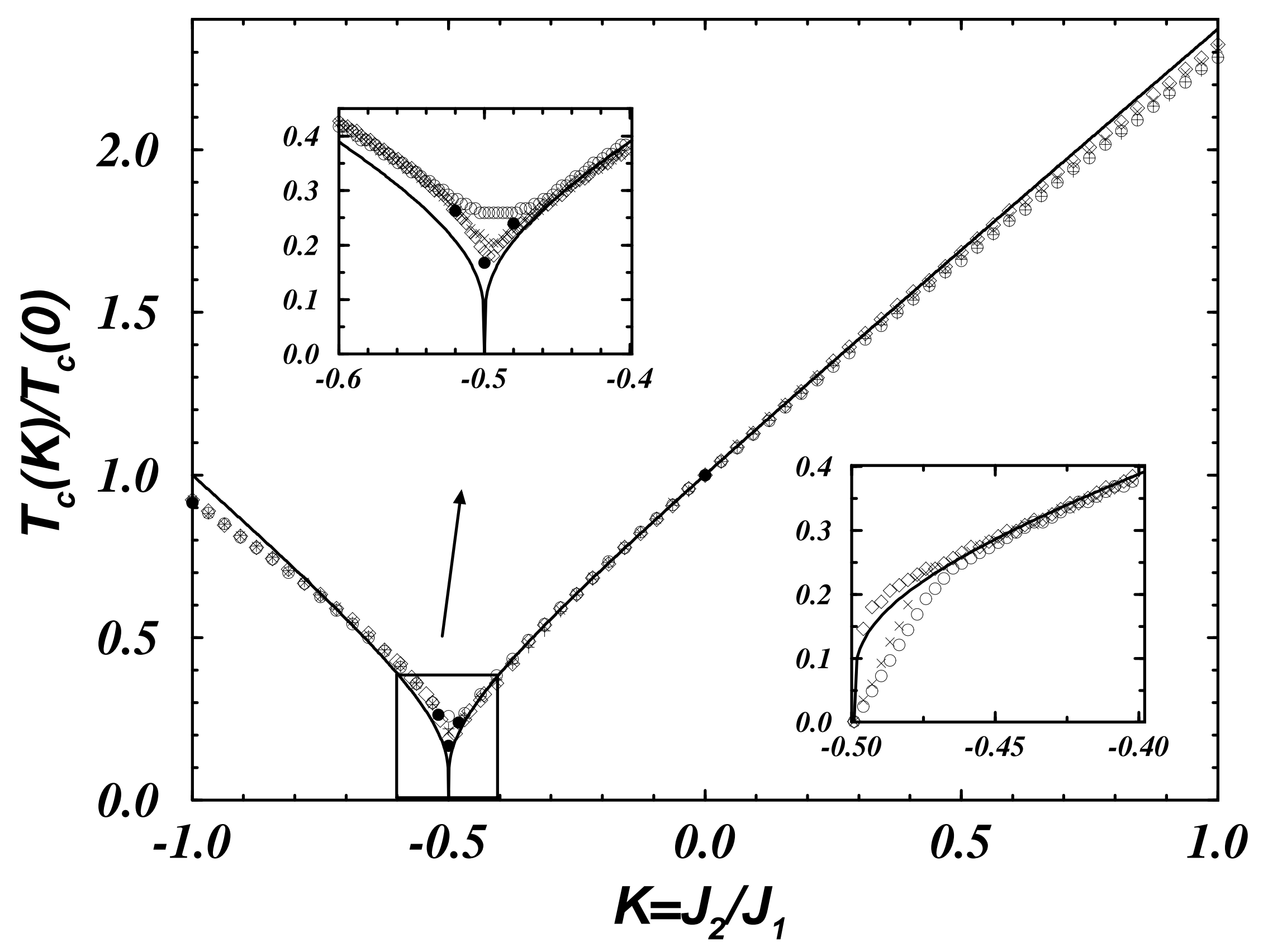




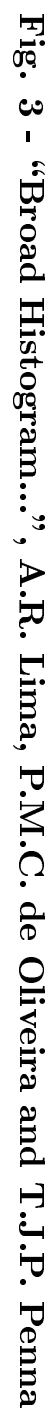

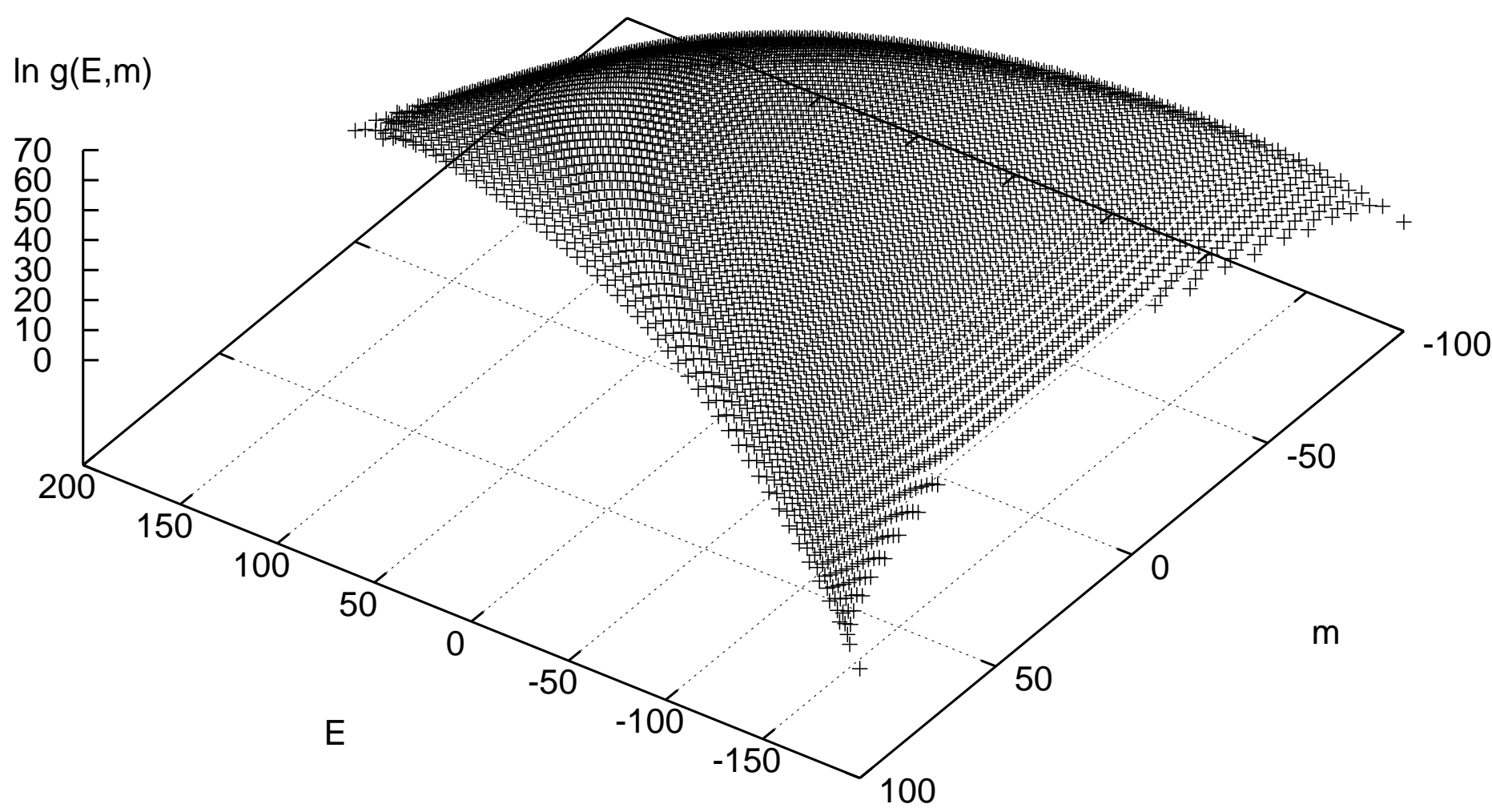


Fig. 4 - "Broad Histogram...", A.R. Lima, P.M.C. de Oliveira and T.J.P. Penna

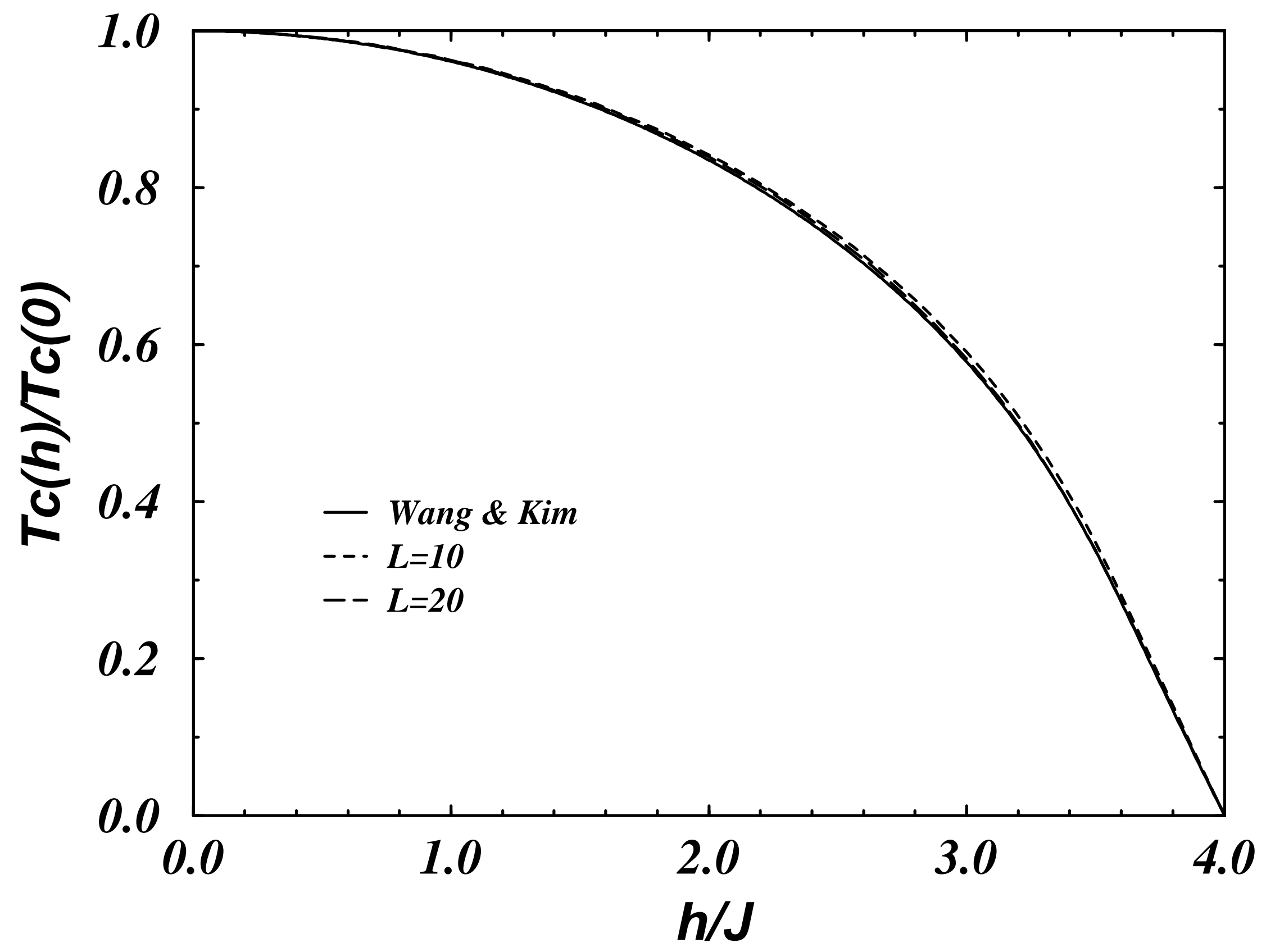

